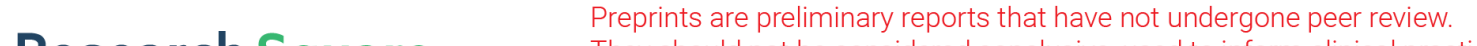 \\ They should not be considered conclusive, used to inform clinical practice, or referenced by the media as validated information. \\ Vaginal Bleeding As The Presenting Manifestation of Post-Hepatectomy/-Splenectomy Portal- Mesenteric Venous Thrombosis
}

\section{Che-Ming Yeh}

Far Eastern Memorial Hospital

Jen-Tang Sun

Far Eastern Memorial Hospital

\section{Chieh-Min Fan}

Far Eastern Memorial Hospital

Kuang-Chau Tsai

Far Eastern Memorial Hospital

Chih-Jung Chang ( $\sim$ chihjung0729@gmail.com )

Far Eastern Memorial Hospital

\section{Case report}

Keywords: Hepatectomy, Mesenteric vein thrombosis, Portal vein thrombosis, Splenectomy, Vaginal bleeding

Posted Date: July 19th, 2021

DOl: https://doi.org/10.21203/rs.3.rs-685240/v1

License: (c) (i) This work is licensed under a Creative Commons Attribution 4.0 International License. Read Full License 


\section{Abstract}

Background: Portal-mesenteric venous thrombosis is not uncommon after hepatectomy or splenectomy but is under-recognized because of non-specific symptoms and lack of awareness of the clinicians. However, misdiagnosis or delayed treatment may have fatal consequences.

Case Presentation: A 57-year-old menopausal woman with the medical history of hepatocellular carcinoma, stage I, underwent laparoscopic partial hepatectomy (S4) and splenectomy 2 months before the presentation of progressive vaginal spotting and vague abdominal pain. Dysfunctional uterine bleeding was suspected initially, but subsequent contrast-enhanced computed tomography due to concern for post-procedure complications revealed thrombosis in the portal vein and superior mesenteric vein. The patient received anticoagulant therapy, and her symptoms gradually resolved.

Conclusions: To the best of our knowledge, vaginal variceal bleeding secondary to portal-mesenteric venous thrombosis has never been reported, but it can be the presenting manifestation for this condition. It should be considered in the differential diagnosis of patients who present with vague abdominal symptoms after hepatectomy or splenectomy.

\section{Background}

Various complications, including portal-mesenteric venous thrombosis (PMVT), liver failure, bile leakage, and intra-abdominal infection, have been reported after hepatectomy or splenectomy. The incidence of PMVT of different etiologies has increased in recent decades due to the increasing use of computed tomography (CT) [1]. Its diagnosis remains clinically challenging owing to the lack of awareness of physicians and non-specific symptoms in patients. Misdiagnosis or delayed treatment can be catastrophic. Herein, we reported a case of PMVT in a patient 2 months after underwent partial hepatectomy and splenectomy who initially presented with vaginal spotting. The patient received anticoagulant therapy and had a good outcome.

\section{Case Presentation}

A 57-year-old woman had a medical history of liver cirrhosis (Child-Pugh score B), complicated with esophageal variceal bleeding, and hepatocellular carcinoma (S4, stage I, pT1bNOMx). She had undergone laparoscopic partial hepatectomy (S4) and splenectomy 2 months before presentation. She visited our emergency department with a 3-day history of progressive vaginal bleeding and vague pain from the periumbilical region to the lower abdomen. She began menopause approximately 10 years ago and had no significant gynecological history. Abdominal pain was mostly aggravated after meals, resulting in poor appetite. She denied having fever, dysuria, hematuria, changes in bowel habits, or bloody or tarry stool.

On arrival, her vital signs were as follows: body temperature, $37.3^{\circ} \mathrm{C}$; respiratory rate, 18 breaths $/ \mathrm{min}$; heart rate, 86 beats/min; and blood pressure, 136/85 mmHg. Physical examination revealed a mildly 
distended abdomen and tenderness in the lower abdominal quadrant without obvious peritoneal signs. Plain radiography showed unremarkable results. Laboratory examinations and urinalysis revealed no obvious abnormalities. Bedside ultrasound was performed, and no ascites, hydronephrosis, or abdominal aortic lesion was found. A gynecologist was consulted for postmenopausal vaginal bleeding, and after pelvic examination and transvaginal sonography, dysfunctional uterine bleeding was suspected.

However, due to concerns regarding post-procedural complications, contrast-enhanced CT was performed, revealing portal vein thrombosis and superior mesenteric vein thrombosis with increased fat stranding around the mesenteric root (Fig. 1). The patient was admitted for treatment using Enoxaparin injection. Vaginal bleeding and abdominal pain gradually resolved, and the anticoagulant was switched to oral Edoxaban 1 week after Enoxaparin injection. The patient was discharged and followed up at the outpatient department uneventfully for one year.

\section{Discussion And Conclusion}

PMVT was first described in 1895 by Elliot [2]. It is an uncommon cause of mesenteric ischemia (6.2\%) but can have fatal consequences, including short bowel syndrome (22.6\%), bowel perforation $(21.0 \%)$, and sepsis (17.0\%) [3]. The overall mortality rate may be as high as $60-80 \%$, especially in patients whose diagnosis is delayed by $>24$ hours after presentation [4]. However, PMVT is not uncommon after hepatectomy and splenectomy, with reported incidence rates of $9.1 \%$ and $4.8 \%$, respectively $[5,6]$. This post-procedure complication is closely related to liver cirrhosis and portal hypertension and has a significantly higher incidence in patients who undergo right hepatectomy and splenectomy and have larger resection volume and longer operation time [7].

The clinical symptoms of PMVT are non-specific, with no direct signs or findings that can confirm the diagnosis in acute or chronic scenarios [8]. Upper gastrointestinal variceal bleeding due to portal hypertension is common, but in some cases, bleeding occurs in the small intestine, colon, or rectum [9]. Vaginal variceal bleeding complicated by portosystemic collateral pathways is very uncommon, and only eight such cases have been reported [10]. A literature review revealed no previous reports of vaginal bleeding owing to PMVT. It has been postulated that the perturbation of the uterine plexus due to previous surgery leads to insufficient decompression of the vaginal venous plexus; this results in shunted blood flow into the systemic circulation, causing vaginal variceal bleeding [10]. However, this phenomenon was not observed in our patient.

Early diagnosis of PMVT is very challenging due to non-specific symptoms, unremarkable findings on physical examination, and generally normal findings on blood tests and plain radiography. Contrastenhanced CT is the diagnostic modality of choice, and treatment should be initiated soon after diagnostic confirmation. Anticoagulants (unfractionated or low-molecular-weight heparin) and thrombolytic agents are the first line of therapy if PMVT is detected $\geq 1$ week post-surgery; otherwise, emergent thrombectomy is strongly recommended [11]. Bowel resection may be performed if delayed diagnosis results in intestinal ischemia, gangrene, or perforation. 
PMVT following hepatectomy and splenectomy is often under-recognized because of non-specific symptoms and lack of awareness among clinicians. "Ectopic" vaginal bleeding secondary to PMVT is rare but should be considered in the differential diagnosis of patients presenting with vague abdominal symptoms after such procedures. While potentially devastating, PMVT usually responds well to anticoagulation therapy if diagnosed in time. A high level of suspicion, physician vigilance according to medical history and clinical symptoms, and proper imaging diagnostics are key in making an early diagnosis.

\section{Abbreviations}

PMVT: Portal-mesenteric venous thrombosis

CT: computed tomography

\section{Declarations}

\section{Ethics approval and consent to participate}

- Not applicable.

\section{Consent for publication}

- Written informed consent was obtained from the patient for publication of this case report and accompanying images.

\section{Availability of data and materials}

- The datasets used and analyzed during the current study are available from the corresponding author on reasonable request.

\section{Competing interests}

- The authors declare that they have no competing interests.

\section{Funding}

- No financial support.

\section{Authors' contributions}

- Che-Ming Yeh was the major contributor in writing the manuscript.

- Chih-Jung Chang reviewed and edited the original draft.

- Supervision: Jen-Tang Sun, Chieh-Min Fan, Kuang-Chau Tsai 
- All authors read and approved the final manuscript.

\section{Acknowledgements}

- We thank the patient and the staff at the Department of Emergency Medicine, Far Eastern Memorial Hospital, for their valuable supports of this report.

\section{References}

1. Acosta S, Alhadad A, Svensson P, Ekberg O. Epidemiology, risk and prognostic factors in mesenteric venous thrombosis. Br J Surg. 2008;95(10):1245-51. https://doi.org/10.1002/bjs.6319.

2. Elliot JW. The operative relief of gangrene of intestine due to occlusion of the mesenteric vessels. Ann Surg. 1895;21:9-23. https://doi.org/10.1097/00000658-189521060-00002.

3. Rhee RY, Gloviczki P, Mendonca CT, Petterson TM, Serry RD, Sarr MG, et al. Mesenteric venous thrombosis: Still a lethal disease in the 1990s. J Vasc Surg. 1994;20:688-97. doi:10.1016/s07415214(94)70155-5.

4. Lewiss RE, Egan DJ, Shreves A. Vascular abdominal emergencies. Emerg Med Clin North Am. 2011;29(2):253 - 72, viii. https://doi.org/10.1016/j.emc.2011.02.001.

5. Yoshiya S, Shirabe K, Nakagawara $\mathrm{H}$, et al. Portal vein thrombosis after hepatectomy. World J Surg. 2014;38(6):1491-7. https://doi.org/10.1007/s00268-013-2440-8.

6. Stamou KM, Toutouzas KG, Kekis PB, Nakos S, Gafou A, Manouras A, et al. Prospective Study of the Incidence and Risk Factors of Postsplenectomy Thrombosis of the Portal, Mesenteric, and Splenic Veins. Arch Surg. 2006;141(7):663-69. doi:10.1001/archsurg.141.7.663.

7. Zhang ZY, Dong KS, Zhang EL, Huang ZY, Chen XP, Dong HH. Acute portal vein thrombosis after hepatectomy in a patient with hepatolithiasis: A case report and review of the literature. Medicine. 2018;97(25):e11174. https://doi.org/ 10.1097/MD.0000000000011174.

8. Cudnik MT, Darbha S, Jones J, Macedo J, Stockton SW, Hiestand BC. The diagnosis of acute mesenteric ischemia: A systematic review and meta-analysis. Acad Emerg Med. 2013;20(11):1087100. https://doi.org/10.1111/acem.12254.

9. Harnik IG, Brandt LJ. Mesenteric venous thrombosis. Vasc Med. 2010;15(5):407-18. https://doi.org/10.1177/1358863X10379673.

10. Harnik I, Golowa Y, Massoumi H. Portal hypertensive vaginal bleeding. Hepatology. 2013;57(5):2085-6. https://doi.org/10.1002/hep.26341.

11. Kuboki S, Shimizu H, Ohtsuka M, Kato A, Yoshitomi H, Furukawa K. Incidence, risk factors, and management options for portal vein thrombosis after hepatectomy: a 14-year, single-center experience. Am J Surg. 2015;210(5):878 - 85 e2. https://doi.org/10.1016/j.amjsurg.2014.11.021.

\section{Figures}



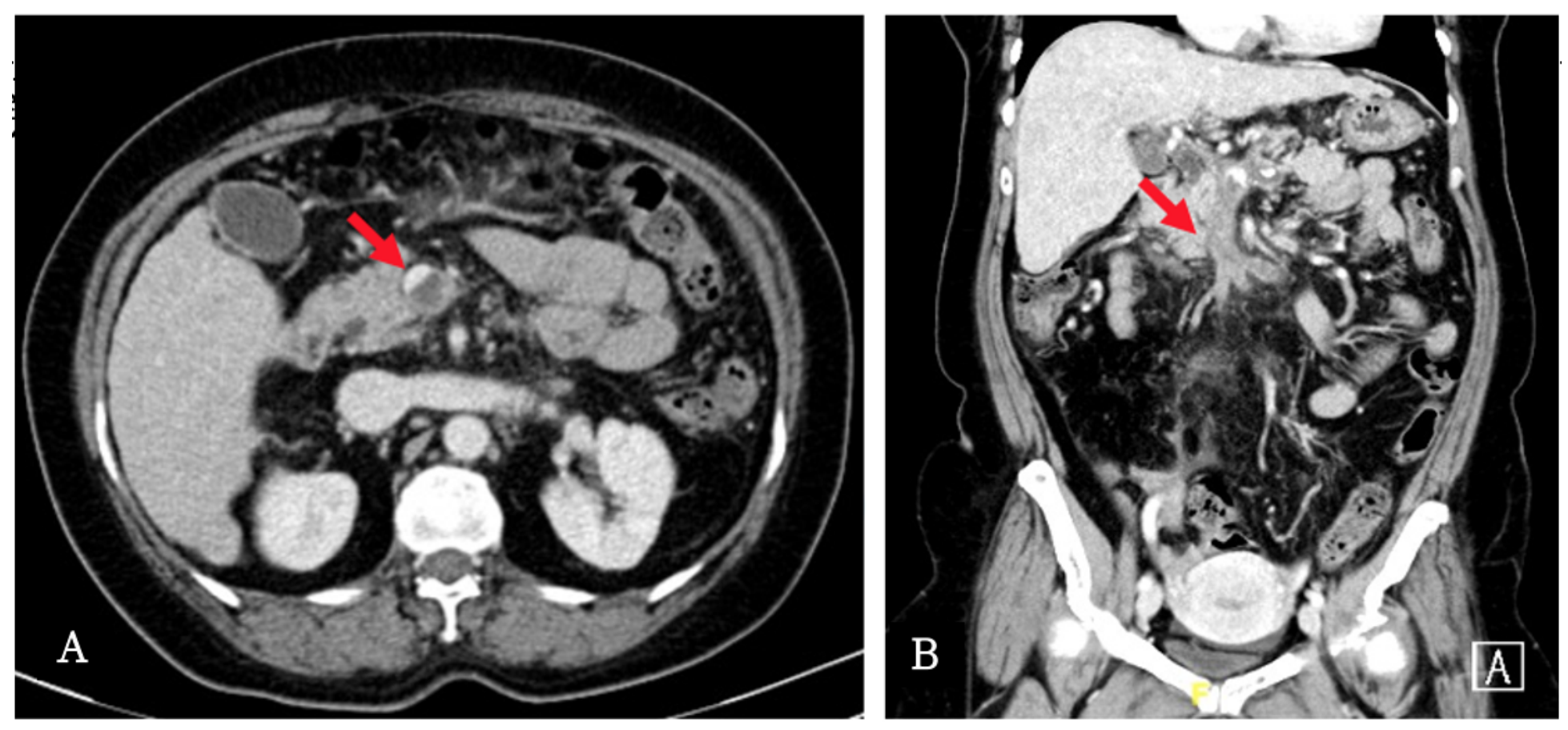

\section{Figure 1}

Contrast-enhanced computed tomography of the abdomen in axial view (A) and coronal view (B), demonstrating thrombus in the portal vein and superior mesenteric vein (red arrow), with surrounding fat stranding. 